\title{
Long term effects of olfactory training in patients with post-infectious olfactory loss*
}

\author{
I. Konstantinidis, E. Tsakiropoulou, J. Constantinidis
}

Smell \& Taste Clinic, 2nd Academic ORL Department, Aristotle University, Thessaloniki, Greece
Rhinology 54: 170-175, 2016

DOI:10.4193/Rhino15.264

*Received for publication:

September 1, 2015

Accepted: October 26, 2015

\begin{abstract}
Background: There is evidence of the effectiveness of repeated exposure to odours on short-term olfactory function. The aim of this study was to assess the long-term effects of olfactory training.

Methods: We conducted a prospective study of 111 patients with post-infectious olfactory dysfunction. Two groups of patients performed olfactory training for 16 and 56 weeks, respectively, and were compared with a control group. The training was performed twice daily using four odours (phenyl ethyl alcohol, eucalyptol, citronellal, eugenol). Olfactory testing was performed by means of the "Sniffin' Sticks" test as a baseline assessment and then every 8 weeks for 56 weeks. Subjective ratings were performed using a visual analogue scale (0-100).
\end{abstract}

Results: Both training groups presented significantly higher scores than the controls. The long-term group had better results than the short-term group. Short-term training patients sustained their improvement within the follow-up period. Subsets analysis showed that training patients mainly increased identification and discrimination. Subjective ratings were in accordance with the olfactory test results.

Conclusion: Long-term olfactory training seems to be associated with better results in patients with post-infectious olfactory loss than a short-term scheme. Short-term training provides sustainable results at 56 weeks follow-up assessment.

Key words: anosmia, post-infectious, olfactory training, long term results

\section{Introduction}

Infections of the upper respiratory tract (URTIs) are one of the most frequent causes of olfactory dysfunction affecting almost 1 in 3 patients visiting a smell and taste clinic ${ }^{(1-5)}$. Fortunately, these patients present a relatively high rate of spontaneous recovery, with about one-third exhibiting improvement of olfactory function one year after the initial assessment ${ }^{(1)}$. Although many medications have been used as treatment modalities none of them proved to be effective ${ }^{(6)}$. However, a new treatment option based on repeated exposure to odours, called olfactory training, offered the most promising results. First, Hummel et al. showed that a structured, short-term exposure to odorants in a 12 weeks study increased olfactory function ${ }^{(7)}$. Additional studies on postinfectious patients (post-URTI) showed a clear benefit from the olfactory training. Specifically, in a previous study we reported 81 post-URTI patients undergoing 16 weeks of training who had clearly better results than controls (2/3 of training patients improved vs $1 / 3$ of controls) ${ }^{(8)}$. Furthermore, a multicentric randomized controlled study from Germany established the positive effect of olfactory training in post-URTI patients after 36 weeks of training ${ }^{(9)}$. Although there is a growing body of literature on the effectiveness of olfactory training even with modified forms 
(10), many questions remain about the sustainability of its results and the optimal period of training required.

This study assesses the kinetics of the olfactory function in patients who had a short-term training in comparison with a group of patients having long-term training and a group of patients with spontaneous recovery.

\section{Materials and methods}

Patients

All patients enrolled in this study were assessed at the Smell \& Taste Clinic of the 2nd Department of Otorhinolaryngology, Aristotle University of Thessaloniki Medical School within a period of six years.

A detailed clinical evaluation including nasal endoscopy was performed in all patients. Depending on the clinical findings and a structured history designed for smell disorders, only postURTI patients were selected for the present study. Patients with chronic rhinosinusitis with or without polyps, post-traumatic or idiopathic olfactory dysfunction were excluded. Diagnosis of a neurological disorder such as Alzheimer's and Parkinson's disease was also an exclusion criteria.

In all patients age, gender, duration of the disease and presence of parosmia and/or phantosmia were recorded.

The olfactory training procedure was explained in details to all patients and they had to decide between following the training scheme or to wait for spontaneous recovery. Patients who preferred the training scheme were randomly divided into two groups: 1) patients with olfactory training for 16 weeks and 2) patients with olfactory training for 56 weeks. The third group included those who decided to wait for spontaneous recovery (control group). All patients who followed the training scheme provided a signed informed-consent according to the study protocol based on Helsinki Declaration for human subjects (JAMA 2000; 284:3043-3049). The study design had the approval of the ethic committee of the Medical Faculty of the Aristotle University of Thessaloniki.

\section{Olfactory testing}

Clinical testing of olfactory function included the Greek verbal version of the "Sniffin' Sticks" battery test ${ }^{(11,12)}$. The test assesses odour threshold, discrimination and identification, and a total score (TDI) was recorded for each patient at the initial appointment. Then all patients of the three study groups were tested every 8 weeks at fixed follow-up appointments for a period of 56 weeks.

A TDI score less than 16.5 corresponds to functional anosmia, a score between 16.5 and 30.5 is considered as hyposmia and a TDI score more than 30.5 as normal olfactory function ${ }^{(11)}$. According to previous studies, an increase in TDI score of $\geq 6$ can be considered as an improvement of olfactory function with a clinical value ${ }^{(13)}$.
Subjective ratings of olfactory function were also recorded at baseline and follow-up appointments by means of a visual analogue scale, scoring from 0 to 100 . On this scale, 0 represents complete olfactory loss and 100 perfect olfactory function. In addition, patients were asked about the presence of qualitative olfactory disorders such as parosmia and phantosmia.

\section{Olfactory training}

Olfactory training included four odorants as representatives of four basic odour categories described by Henning: flowery, fruity, aromatic and resinous ${ }^{(14)}$. Specifically, patients were exposed twice daily to four odours: phenyl ethyl alcohol (PEA): rose, eucalyptol: eucalyptus, citronellal: lemon, and eugenol: cloves, according to the scheme described by Hummel et al. ${ }^{(7)}$. This includes exposure to odorants twice a day for 5 minutes. Every 5 minute session consisted of rotated exposure to each odorant for 10 seconds, with time intervals of 10 seconds between odours. Patients were advised to perform the training procedure at a standard moment in the morning and evening. Patients of the training groups who discontinued the scheme for $\geq 7$ days were excluded from the study. Patients in the control group did not follow any pharmaceutical or alternative treatment modalities.

\section{Statistical analysis}

Statistical analysis was performed using SPSS (Statistical Packages for Social Sciences, version 20.0, SPSS Inc., Chicago, IL, USA). Demographic and clinical data are presented as means \pm standard deviation (SD) or percentages (\%). Comparisons between the training groups and controls were performed using the t-test for independent samples and the $x^{2}$-test. A multivariate ANOVA analysis using the general linear model was performed to assessment the olfactory function in relation with the continuous variables measured in this study.

The alpha level for all statistical tests was set at $p=0.05$. Correlation analyses were performed according to Pearson. Bonferroni correction was used for post-hoc analyses.

\section{Results}

\section{Participants}

In total 111 patients out of 116 were included in this study during a 6-year study period (42 male/ 69 female, mean age 62.9 \pm 6.2 y.o, range $25-76$ y.o.). The short-term group consisted of 36 patients and the long-term group of 34 training patients and the control group of 41 patients. Five patients discontinued the training scheme ( 2 in the short-term group, 3 in the long-term group) and were excluded from the study (total discontinuation rate: $5 / 75,6.6 \%$ ). Three of them reported irritation of the nose and headache after odorant exposure and two patients were unable to comply with the long treatment process. At baseline assessment, both the training and non-training 
Table 1. Demographics and descriptive statistics (means \pm SD) of the three study groups at baseline and weeks 16 and 56. ( $p$ values are presented as > or < indicating all the possible comparisons between groups. One asterisk indicates significant differences between the two training groups and controls, two asterisks indicate an additional significant difference between training groups).

\begin{tabular}{|c|c|c|c|c|}
\hline & $\begin{array}{l}\text { Short-term } \\
\qquad n=36\end{array}$ & $\begin{array}{l}\text { Long-term } \\
\qquad n=34\end{array}$ & $\begin{array}{c}\text { Control } \\
n=41\end{array}$ & p-values \\
\hline Age (years) & $61.5 \pm 6.5$ & $62.2 \pm 5.9$ & $60.1 \pm 5.8$ & $p>0.2$ \\
\hline Gender (n) & $14 m / 22 f$ & $13 \mathrm{~m} / 21 \mathrm{f}$ & $15 \mathrm{~m} / 26 \mathrm{f}$ & $p>0.2$ \\
\hline $\begin{array}{l}\text { Duration of } \\
\text { disease (in } \\
\text { months) }\end{array}$ & $9.1 \pm 3.1$ & $9.5 \pm 3.2$ & $8.9 \pm 2.8$ & $p>0.7$ \\
\hline TDI baseline & $15 \pm 2.2$ & $15.9 \pm 2.2$ & $15.2 \pm 1.8$ & $p>0.7$ \\
\hline TDI 16weeks & $23,51 \pm 2,1$ & $23.52 \pm 1.8$ & $16.5 \pm 2.4$ & $p<0.03^{*}$ \\
\hline TDI 56weeks & $24.1 \pm 1,5$ & $27.3 \pm 1,5$ & $20.5 \pm 1,6$ & $\mathrm{p}<0.05^{* *}$ \\
\hline $\begin{array}{l}\text { Identification } \\
\text { baseline }\end{array}$ & $7.5 \pm 1.5$ & $8.1 \pm 1.5$ & $7.3 \pm 1.7$ & $p>0.7$ \\
\hline $\begin{array}{l}\text { Identification } \\
16 \text { weeks }\end{array}$ & 11.9. \pm 1.3 & $12.5 \pm 1.2$ & $7.5 \pm 1.4$ & $\mathrm{p}<0.05^{*}$ \\
\hline $\begin{array}{l}\text { Identification } \\
56 \text { weeks }\end{array}$ & $12.2 \pm 1.2$ & $14.4 \pm 1.2$ & $9.6 \pm 1.4$ & $\mathrm{p}<0.05^{*}$ \\
\hline $\begin{array}{l}\text { Threshold } \\
\text { baseline }\end{array}$ & $2.2 \pm 1.2$ & $2.1 \pm 1.2$ & $1.9 \pm 1$ & $p>0.8$ \\
\hline $\begin{array}{l}\text { Threshold } \\
16 \text { weeks }\end{array}$ & $2.4 \pm 1.3$ & $2.4 \pm 1.2$ & $2.1 \pm 1.3$ & $p>0.9$ \\
\hline $\begin{array}{l}\text { Threshold } \\
56 \text { weeks }\end{array}$ & $2.3 \pm 1.3$ & $2.7 \pm 1.3$ & $2.2 \pm 1.3$ & $p>0.6$ \\
\hline $\begin{array}{l}\text { Discrimination } \\
\text { baseline }\end{array}$ & $5.5 \pm 1.8$ & $5.3 \pm 1.4$ & $5.7 \pm 1.5$ & $p>0.7$ \\
\hline $\begin{array}{l}\text { Discrimination } \\
16 \text { weeks }\end{array}$ & $9.2 \pm 1.1$ & $9.1 \pm 1.2$ & $6.9 \pm 1.4$ & $p>0.06$ \\
\hline $\begin{array}{l}\text { Discrimination } \\
56 \text { weeks }\end{array}$ & $9.8 \pm 1.2$ & $10.2 \pm 0.9$ & $8.7 \pm 1.1$ & $p>0.06$ \\
\hline $\begin{array}{l}\text { Subjective } \\
\text { ratings baseline }\end{array}$ & $20.5 \pm 4.2$ & $22.3 \pm 5$ & $19.2 \pm 4.8$ & $p>0.7$ \\
\hline $\begin{array}{l}\text { Subjective ra- } \\
\text { tings } 16 \text { weeks }\end{array}$ & $45.3 \pm 4.1$ & $47.7 \pm 4.8$ & $23.5 \pm 4.6$ & $\mathrm{p}<0.01^{*}$ \\
\hline $\begin{array}{l}\text { Subjective ra- } \\
\text { tings } 56 \text { weeks }\end{array}$ & $38 \pm 4.5$ & $55.4 \pm 4.7$ & $28.4 \pm 3.8$ & $\mathrm{p}<0.03^{* *}$ \\
\hline
\end{tabular}

patients had similar age and sex distribution. In addition, at the time of diagnosis the three study groups did not differ significantly in the olfactory test results $(F[2,108]<1.91, p>0.2)$. Demographics of all groups are presented in Table 1.

\section{Comparison of psychophysics, short term training vs long term training vs controls}

Comparison of TDI score differences between baseline assessment and after 56 weeks demonstrated that both training groups together had significantly better olfactory test results

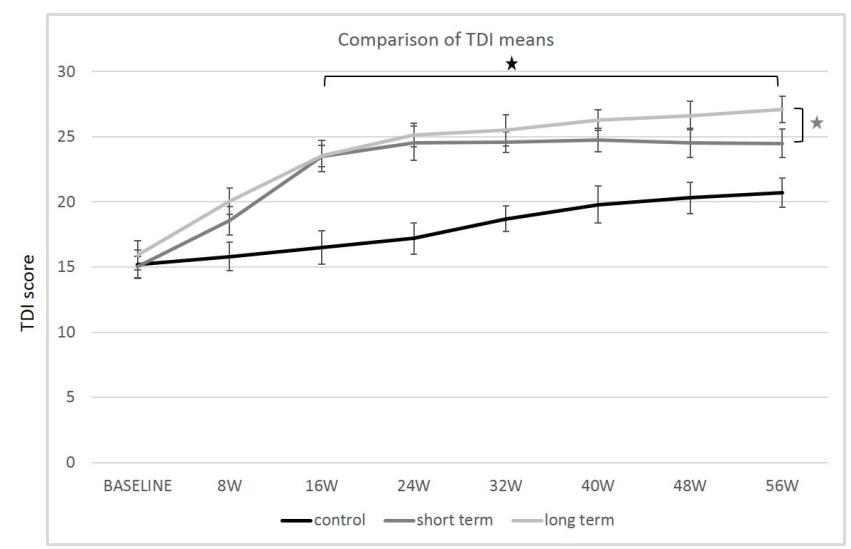

Figure 1. Comparison of TDI means \pm SD between the three study groups across the follow up period. The black asterisk indicates the significant difference within the long-term group between the TDI means of 16 th and 56th weeks. The grey asterisk indicates the only significant difference noticed between short-term and long-term training groups across the study period.

than controls showing a positive effect of training on olfaction $(\mathrm{F}[2,108]=10.54, \mathrm{p}=0.005)$.

Comparison of TDI-means showed that patients in the long-term training group after the initial 16 weeks of training had better results than those of the short-term group at all follow-up appointments. However, none of these differences were significant with the exception of the last appointment in week $56(p=$ 0.038; Figure 1). Subtest analyses showed significant differences in odour identification and a trend towards significance in odour discrimination between both training groups and the control group. However, this was not the case for the odour threshold (Table 1).

Both training groups showed a better improvement period within the first 16 weeks. The short-term training patients who discontinued this scheme sustained the same olfactory test results and the long-term training patients showed a slower increase of their olfactory function untill the end of the followup period. Specifically, the latter group presented a $70.9 \%$ of the total TDI-score improvement within the first 16 weeks and only $29,1 \%$ within the last 40 weeks ( $X^{2}$ test: $p=0.02$ ).

In contrast, patients in the control group showed a more linear improvement in their olfactory test results (Figure 1). Comparison of TDI-means within training groups showed significant differences in both groups from baseline assessment to 8 weeks, between 8 weeks and 16 weeks (all $p<0.01$ ) and in the long-term group only between 16 weeks and 56 weeks ( $p=$ 0.035).

Analysis of the distribution of patient's in "improved", "no change" and "worse" olfactory function after training or not (Figure 2) showed that the long-term group had not only better olfactory test results but also a higher number of improved 

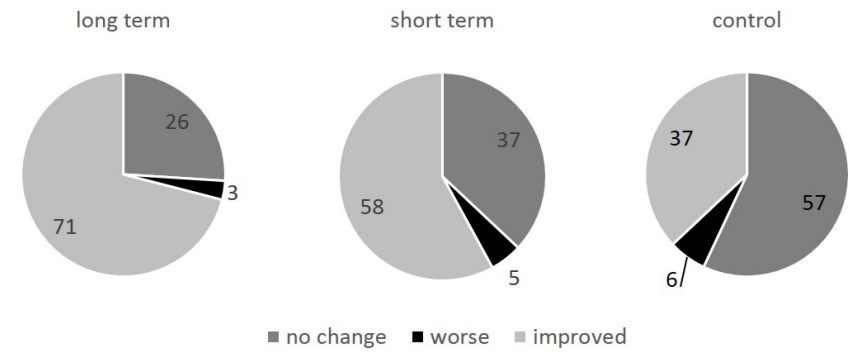

Figure 2. Percentages of individual improvement, no change or worsening within the study groups (change in the baseline TDI score of clinical value at 56 weeks assessment was considered that of $\geq 6$ ).

patients (improved patients long-term $71 \%$ vs short-term $58 \%$, $x^{2}$ test: $p=0.068$ ). Interestingly, three of the patients in the longterm group presented a late response to olfactory training with their main improvement occurring during weeks $24-32$ of the treatment.

\section{Gender and age}

Gender had no significant effect on olfactory recovery in either training groups or controls. Comparison of the mean TDI improvement between males and females showed no statistical significance (long-term training: $p=0.41$ short-term training: $p=0.38$, controls: $p=0.31$ ). In a similar way, the factor age did not have any effect on olfactory function improvement. No correlation was found between a TDI change of $\geq 6$ and age of the patients, with only a trend towards a significant negative correlation in the control group $(r=-0.18, p=0.09)$.

\section{Severity and duration of olfactory dysfunction}

The severity of the olfactory impairment (anosmia / hyposmia according to olfactory testing) at the initial assessment did not correlate with the improvement of olfactory function of patients in either training groups at any of the follow-up measurements (all $r<0.11$ ). No difference in the pattern of improvement in training patients between hyposmics and anosmics was seen (Figure 3).

In both training groups, patients with a shorter duration of olfactory loss had a higher chance to improve their overall olfactory function (long-term: $r=-0.29 ; p=0.007$, short-term: $r=-0.25$; $p=0.009)$. The same effect was shown in controls $(r=-0.28$; $\mathrm{p}=0.011$ ).

\section{Subjective ratings}

Analysis of subjective rating means in both training groups showed that the training patients at all follow-up appointments rated their olfactory function significantly higher than the controls. Training patients presented a significant increase at every appointment untill 24 weeks in comparison with the previous

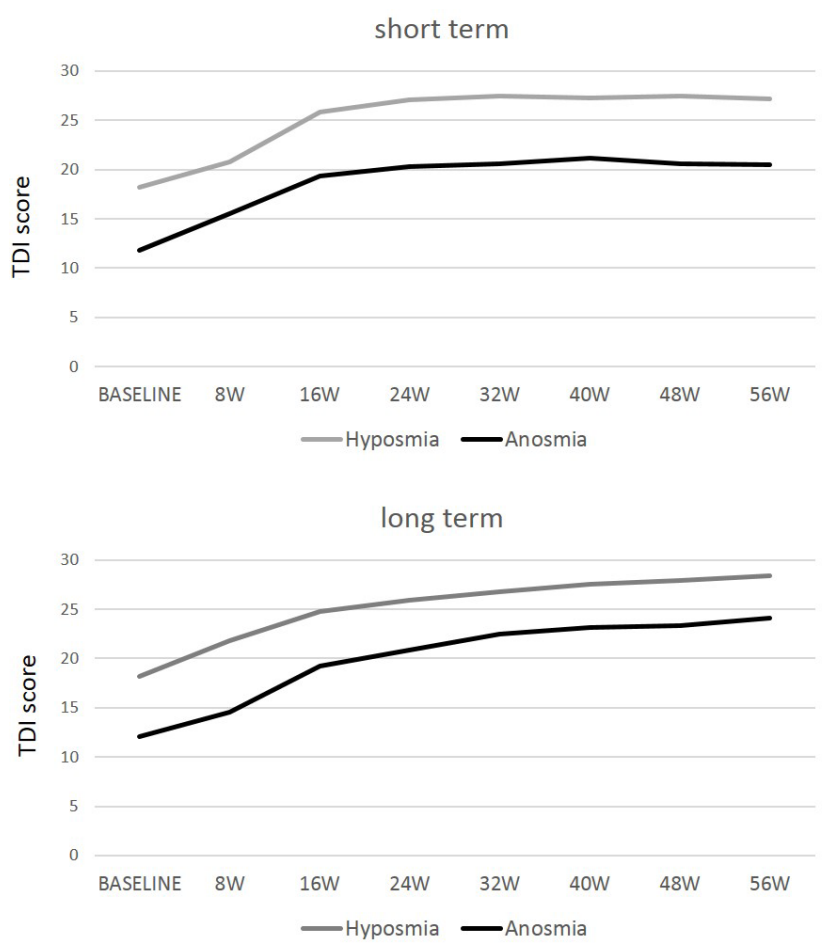

Figure 3. Comparison of TDI means between hyposmics and anosmics (according to their baseline TDI score) in both olfactory training groups revealed a similar pattern of recovery.

one (all $\mathrm{p}<0.05$ ). At subsequent visits, no further significant change was reported. A trend towards significance was found in short-term training patients with lower ratings after 56 weeks compared with their ratings after 16 weeks of training $(p=0.07)$.

\section{Qualitative disorders}

Parosmia and/or phantosmia as reported by the patients at the initial assessment was not correlated with the improvement in TDI total score or any of the subtests score in all study groups (all $r<0.1$ ).

\section{Discussion}

Evidence exists from recent publications that olfactory training can be used as a treatment option for post-URTI patients ${ }^{(7-10)}$. However, many questions remain regarding the appropriate duration of olfactory training and its long-term effect. The present study provides the longest follow-up in the literature thus far on olfactory training.

Our data analysis shows two main results:

1. Olfactory training even for a short period ( 16 weeks) provides relatively sustainable results lasting 56 weeks.

2. An extended period of training ( 56 weeks) provides further olfactory improvement, however, the degree of this improvement is significantly less after the initial 16 weeks of training. 
Short-term olfactory training provides a sustainable improvement of at least 56 weeks, which is significantly higher than that of the control group. This is important as it provides evidence that the training effect is not only an acceleration of the natural history of the disease, but also modulates the olfactory system in a permanent way possibly through central neuronal changes. A recent $\mathrm{f}-\mathrm{MRI}$ study on patients with post-infectious olfactory loss revealed that short-term olfactory training (12 weeks) induced alterations in functional connectivity networks ${ }^{(15)}$. The authors stated that olfactory training is capable of inducing neuronal reorganization processes. This statement was based on the observation that a diverse network between the pyriform cortex and mostly non-olfactory regions shown before the olfactory training significantly declined after the training ${ }^{(15)}$.

These central changes are in accordance with clinical data where olfactory training was found to affect mainly odour identification and discrimination reflecting higher cognitive functions, and not an olfactory threshold, which is more related with peripheral changes ${ }^{(8-10)}$. However, a positive effect of training on the peripheral olfactory system cannot be excluded as proposed in other studies ${ }^{(15,16)}$. For example, Wang et al., demonstrated a peripheral plasticity of olfactory system of human adults after repeated exposure in androstenone as measured by an increase in electro-olfactogram (potentials from the olfactory epithelium) and a decreased detection threshold in olfactory testing ${ }^{(16)}$. An extended period of training provides higher olfactory results, however, in a non-linear way and mostly within the first 16 weeks. As olfactory function continues to improve in a different way, it seems possible that two different processes exist: an initial fast phase ( 16 weeks) where the main clinical improvement occurs while imaging findings suggest that non-olfactory functional connections have declined. A second slower phase seems to follow where the clinical improvement is not so profound and a different neuronal reorganization may be in progress. At the moment the maximum duration of an olfactory training period remains unclear and its central, and peripheral effects have not been studied through later imaging modalities. On the contrary, the control group presented a more linear natural history of the disease in accordance with previous studies ${ }^{(9,17)}$.

Imaging studies reported that mainly grey and white matter volume decreases in anosmic patients compared to healthy controls, and the degree of this atrophy is correlated with longer disease duration ${ }^{(18,19)}$. This volume loss was detected in olfactory areas, such as the pyriform cortices, but also in brain areas with more generalized function, such as the anterior cingulate cortex or the anterior insular cortex. A significant observation was that patients with more than a one year history of the disease had significantly more atrophy than those with a shorter duration ${ }^{(19)}$.
This atrophy process seems to become more serious as anosmia progresses and may require longer treatment with olfactory training to restore central connections and regain brain volume loss, especially in patients with long history of the disease. An early, rapid improvement that can be experienced subjectively is important for a long-term treatment programme. This is because patients tend to follow it more easily when they recognize the benefit at a relatively early stage. However, patients should not be disappointed if no change is detected at this early timepoint as a small percentage of them may experience improvement after 6 or 8 months of training as seen in our study. In all studies, olfactory training is based on active sniffing of odorants and thus we cannot distinguish the possible positive role that sniffing itself might have on olfactory recovery. However, Damm et al, provided data from a group trained with a super-low concentration very close to a non-odour condition suggesting that sniffing itself does not play a significant role ${ }^{(9)}$. Another limitation of our research causing bias is that it is not a placebo controlled study and patients in the control group knew that they did not receive any specific treatment. However, the use of odorless liquids for a placebo control group can be easily detected by the patient and thus we decided not to follow this procedure.

In this study, we relied on the patients' statement every two months regarding their discipline to follow the long-term treatment. This undoubtedly poses questionsas to whether patients really strictly followed the daily programme. Further studies are needed for the standardization of the procedure in a way that will confirm the compliance of patients and will improve their follow-up.

\section{Conclusion}

Short-term olfactory training results in sustainable olfactory improvement greater than that of the natural course of the disease aover a 56 weeks follow-up assessment. Long-term olfactory training presented further improvement of olfactory function within this period with a first fast recovery period of 16 weeks and a second slower period of 40 weeks.

\section{Authorship contribution}

IK: study design, data analysis, writting of manuscript; ET: data collection, manuscript corrections; JC: manuscript corrections, study overview

\section{Conflict of interest}

All authors declare that they have no financial interest for the presented research.

\section{References}

1. Seiden AM. Postviral olfactory loss.
Otolaryngol Clin North Am 2004; 37:11591166.
2. Hummel T, Nordin S. Olfactory disorders and their consequences for quality of 
life--a review. Acta Oto-Laryngol 2005; 125:116-121.

3. Doty RL. The olfactory system and its disorders. Semin Neurol 2009; 29: 74-81.

4. Holbrook EH, Leopold DA. An updated review of clinical olfaction. Curr Opin Otolaryngol Head Neck Surg 2006; 14 23-28.

5. Damm M, Temmel A, Welge-Lussen A, et al. Olfactory dysfunctions: epidemiology and therapy in Germany, Austria and Switzerland. HNO 2004; 52: 112-120.

6. Reden J, Herting B, Lill K, Kern R, Hummel T. Treatment of postinfectious olfactory disorders with minocycline: a double-blind, placebo-controlled study. Laryngoscope 2011;121:679-682.

7. Hummel T, Rissom K, Reden J, Hähner A Weidenbecher M, Hüttenbrink KB. Effects of olfactory training in patients with olfactory loss. Laryngoscope 2009; 119: 496-499.

8. Konstantinidis I, Tsakiropoulou E, Bekiaridou P, Kazantzidou C, Constantinidis J. Use of olfactory training in posttraumatic and postinfectious olfactory dysfunction. Laryngoscope 2013; 123: 85-90.

9. Damm M, Pikart LK, Reimann $\mathrm{H}$ et al. Olfactory training is helpful in postinfectious olfactory loss: a randomized, controlled, multicenter study. Laryngoscope
2014; 124: 826-831.

10. Altundag A, Cayonu M, Kayabasoglu G et al. Modified olfactory training in patients with post-infectious olfactory loss. Laryngoscope 2015; 125:1763-1766.

11. Hummel T, Kobal G, Gudziol H, Mackay$\operatorname{Sim}$ A. Normative data for the"Sniffin' Sticks" including tests of odor identification, odor discrimination, and olfactory thresholds: an upgrade based on a group of more than 3,000 subjects. Eur Arch Otorhinolaryngol 2007: 264:237-243.

12. Konstantinidis I, Printza A, Genetzaki S, Mamali K, Kekes G, Constantinidis J. Cultural adaptation of an olfactory identification test:the Greek version of Sniffin' Sticks. Rhinology 2008; 46: 292-296.

13. Gudziol V, Lötsch J, Hähner A, Zahnert T, Hummel T. Clinical significance of results from olfactory testing. Laryngoscope 2006; 116: 1858-1863.

14. Henning H. Der Geruch. Leipzig, Germany: Johann Ambrosius Barth; 1916.

15. Kollndorfer K, Kowalczyk K, Hoche E et al Recovery of olfactory function induces neuroplasticity effects in patients with smell loss. Neural Plast 2014; 2014:140419.

16. Wang L, Chen L, Jacob T. Evidence for peripheral plasticity in human odour response. J Physiol 2004; 554: 236-244.
17. Reden J, Mueller A, Mueller C et al. Recovery of olfactory function following closed head injury or infections of the upper respiratory tract. Arch Otolaryngol Head Neck Surg 2006; 132: 265-269.

18. Bitter T, Gudziol H, Burmeister HP, Mentzel HJ, Guntinas-Lichius O, Gaser C. Anosmia leads to a loss of gray matter in cortical brain areas. Chem Senses 2010; 35: 407415

19. Peng P, Gu H, Xiao W, et al. A voxel-based morphometry study of anosmic patients. $\mathrm{Br}$ J Radiol. 2013; 86: 20130207

\section{Konstantinidis}

3A Kapetan Kotta str

Panorama

Thessaloniki

55523, Greece

Tel: +30-697-322 9525

Fax: +30-231-093 4888

E-mail:jordan_orl@hotmail.com 